\title{
Friedmann-like universes with torsion
}

\author{
Dimitrios Kranas $^{1,4}$, Christos G. Tsagas ${ }^{1,2, a}$, John D. Barrow ${ }^{2}$, Damianos Iosifidis ${ }^{3}$ \\ ${ }^{1}$ Section of Astrophysics, Astronomy and Mechanics, Department of Physics, Aristotle University of Thessaloniki, 54124 Thessaloniki, Greece \\ 2 DAMTP, Centre for Mathematical Sciences, University of Cambridge, Wilberforce Road, Cambridge CB3 OWA, UK \\ ${ }^{3}$ Theoretical Physics Division, Department of Physics, Aristotle University of Thessaloniki, 54124 Thessaloniki, Greece \\ ${ }^{4}$ Present address: Department of Physics and Astronomy, Louisiana State University, Baton Rouge, LA 70803, USA
}

Received: 14 January 2019 / Accepted: 29 March 2019 / Published online: 15 April 2019

(C) The Author(s) 2019

\begin{abstract}
We consider spatially homogeneous and isotropic cosmologies with non-vanishing torsion, which assumes a specific form due to the high symmetry of these universes. Using covariant and metric-based techniques, we derive the torsional versions of the continuity, the Friedmann and the Raychaudhuri equations. These show how torsion can drastically change the standard evolution of the Friedmann models, by playing the role of the spatial curvature or that of the cosmological constant. We find, for example, that torsion alone can lead to exponential expansion and thus make the Einstein-de Sitter universe look like the de Sitter cosmos. Also, by modifying the expansion rate of the early universe, torsion could have affected the primordial abundance of helium-4. We show, in particular, that torsion can reduce the production of primordial helium- 4 , unlike other changes to the standard thermal history of the universe. These theoretical results allow us to impose strong observational bounds on the relative strength of the associated torsion field, confining its ratio to the Hubble rate within the narrow interval $(-0.005813,+0.019370)$ around zero. Finally, turning to static spacetimes, we demonstrate that there exist torsional analogues of the Einstein static universe with all three types of spatial geometry. These models can be stable when the torsion field and the universe's spatial curvature have the appropriate profiles.
\end{abstract}

\section{Introduction}

General relativity advocates a geometrical interpretation of gravity, which ceases being a force and becomes a manifestation of the non-Euclidean geometry of the host spacetime. The theory is founded on the assumption of Riemannian geometry, where deviations from Euclidean flatness are described by the symmetric Levi-Civita connection, namely

\footnotetext{
a e-mail: tsagas@astro.auth.gr
}

by the Christoffel symbols. Nevertheless, there is no fundamental theoretical reason, apart perhaps from simplicity, for making such an assumption. Allowing for a general asymmetric affine connection introduces spacetime torsion and therefore new geometrical degrees of freedom into the system, since there is now an independent torsional field in addition to the metric. The literature contains a number of suggestions for experimentally testing gravitational theories with non-zero torsion (see [1-7] for a representative though incomplete list). As yet, however, there is no experimental or observational evidence to support the existence of spacetime torsion. The main reason is that, typically, the effects of torsion start becoming appreciable at extremely high energy densities. These densities can be achieved only in the deep interior of compact objects, like neutron stars and black holes, or during the very early stages of the universe's expansion. Such environments are still beyond our experimental capabilities.

Torsion does not naturally fit into highly symmetric spacetimes, like the Friedmann-Robertson-Walker (FRW) models of standard cosmology. Given the spatial homogeneity and isotropy of the latter, the allowed torsion field must satisfy a specific profile [8], which falls into the class of the so-called vectorial torsion fields [9-11]. Practically speaking, spacetime torsion and the associated matter spin are fully determined by a scalar function that depends only on time. Such choices allow us to construct and study the torsional analogues of the classic Friedmann universes. In the process we show that, despite the presence of torsion, the high symmetry of the FRW host preserves the symmetry of the associated Ricci curvature tensor, which implies that the corresponding Einstein and energy-momentum tensors are symmetric as well. Then, using both $1+3$ covariant and metric-based techniques, we present the three key formulae monitoring the evolution of these models, namely the analogues of the Friedmann, the Raychaudhuri, and the continuity equations. 
These enable us to "quantify" the torsion input to the total effective energy density of the system, by means of an associated $\Omega$-parameter, as well as its contribution to the kinematic variables of the cosmological models in question, namely to the Hubble and to the deceleration parameters.

Phenomenologically speaking, torsion can play the role of spatial curvature and reproduce the effects of a cosmological constant, or those of dark energy. As a result, torsional cosmologies (with or without matter) can experience accelerated expansion. We find, in particular, that torsion can force the Milne and the Einstein-de Sitter universes into a phase of accelerated expansion analogous to that of their de Sitter counterpart. These examples suggest that a torsion-dominated early universe, or a dust-dominated latetime cosmos with torsion, could go through a phase of accelerated expansion without the need of a cosmological constant, the inflaton field, or dark energy. Analogous effects were reported in [12-16], suggesting that torsional cosmologies might deserve further scrutiny.

Looking for observational signatures of torsion, we find that the latter can affect the outcome of primordial nucleosynthesis, since it changes the expansion rate of the universe. This can be used to put observational constraints on the allowed torsion fields. Here, we are able to calculate the torsion effect on the amount of helium-4 produced during primordial nucleosynthesis. Combining our theoretical result with the currently allowed range of the primordial helium-4 abundance, leads to a very strong constraint on the strength of the associated torsion field. In particular, the relative torsion contribution to the volume expansion of the universe is found to lie within the narrow interval $(-0.005813,+0.019370)$ around zero.

Finally, we turn our attention to static spacetimes with torsion. In particular, we study the structure of the torsional analogue of the Einstein-static universe and investigate its linear stability. We find that there exist static models with all three types of spatial geometry, that is Euclidean, spherical or hyperbolic. Our last step is to use standard perturbative techniques to test the linear stability of these new spacetimes. We show that static solutions with positive curvature are unstable, while those with zero or negative 3 -curvature can achieve stable configurations.

\section{Spacetimes with torsion}

Riemannian geometry demands the symmetry of the affine connection, thus ensuring torsion-free spaces. Nevertheless, by treating torsion as an independent geometrical field, in addition to the metric, one extends the possibilities to the so-called Riemann-Cartan spaces.

\subsection{Torsion and contortion}

The torsion tensor $\left(S^{a}{ }_{b c}\right)$ of a general spacetime coincides with the antisymmetric component of the affine connection, namely $S^{a}{ }_{b c}=\Gamma^{a}{ }_{[b c]}$. Imposing the familiar metricity condition, that is demanding that the metric tensor is covariantly constant (i.e. $\nabla_{c} g_{a b}=0$ ), splits a generalised (asymmetric) connection as $\Gamma^{a} b c=\tilde{\Gamma}^{a}{ }_{b c}+K^{a} b c$. Here, $\tilde{\Gamma}^{a} b c$ defines the Christoffel symbols and $K^{a} b c$ is the contortion tensor given by $^{1}$

$K_{a b c}=S_{a b c}+S_{b c a}+S_{c b a}=S_{a b c}+2 S_{(b c) a}$,

with $K_{a b c}=K_{[a b] c}$. From the geometrical point of view, torsion prevents infinitesimal parallelograms from closing (e.g. see $[18,19])$. Physically, torsion provides a link between the intrinsic angular momentum (i.e. the spin) of the matter and the geometry of the host spacetime.

The antisymmetry of $S^{a} b c$ guarantees that it has only one non-trivial contraction, leading to the torsion vector

$S_{a}=S_{a b}^{b}=-S_{b a}^{b}$.

As we will see later, the torsion vector becomes the sole carrier of the torsion effects in spatially homogeneous and isotropic spacetimes. Following (1), there is only one independent contraction of the contortion tensor as well. In particular, we have $K_{a b}^{b}=2 S_{a}=-K_{a b}{ }^{b}$ with $K^{b}{ }_{b a}=0$.

\subsection{Field equations and Bianchi identities}

In spacetimes with non-zero torsion, matter and curvature are coupled together by means of the Einstein-Cartan field equations, namely

$R_{a b}-\frac{1}{2} R g_{a b}=\kappa T_{a b}-\Lambda g_{a b}$,

where $R_{a b}$ is the Ricci tensor, $R=R^{a}{ }_{a}$ is the associated scalar and $T_{a b}$ is the energy-momentum tensor of the matter. Note that $R=4 \Lambda-\kappa T$, where $T=T^{a}{ }_{a}$ and $\kappa=8 \pi G$. Although expression (3) is formally identical to its general relativistic counterpart, here both $R_{a b}$ and $T_{a b}$ are generally asymmetric (i.e. $R_{[a b]} \neq 0$ and $T_{[a b]} \neq 0$ ) due to the presence of torsion. The latter is typically coupled to the spin of the matter via the Cartan field equations

$S_{a b c}=-\frac{1}{4} \kappa\left(2 s_{b c a}+g_{c a} s_{b}-g_{a b} s_{c}\right)$,

\footnotetext{
${ }^{1}$ In the literature the definitions of the torsion and the contortion tensors vary. In this study, we have adopted the conventions of [17], which follow those of $[12,13]$, though in the latter the metric signature is $(+,-,-,-)$. Also note that the tildas will always indicate purely Riemannian (torsion free) variables.
} 
with $s_{a b c}=s_{[a b] c}$ and $s_{a}=s^{b}{ }_{a b}$ being the spin tensor and the spin vector respectively. The trace of (4) gives $S_{a}=-\kappa s_{a} / 4$, relating the torsion and the spin vectors directly.

In the presence of torsion, the Bianchi identities acquire a non-zero right-hand side, when compared to their Riemannian analogues. More specifically, we have

$\left.\nabla_{[e} R^{a b}{ }_{c d]}=2 R^{a b}{ }_{f[e} S^{f}{ }_{c d}\right]$

and

$R^{a}{ }_{[b c d]}=-2 \nabla_{[b} S_{c d]}^{a}+4 S^{a}{ }_{e[b} S^{e}{ }_{c d]}$,

where $R_{a b c d}$ is the curvature tensor (with $R_{a b c d}=R_{[a b][c d]}$ only). Contracting the Bianchi identities twice leads to

$\nabla^{b} G_{b a}=2 R_{b c} S^{c b}{ }_{a}+R_{b c d a} S^{d c b}$

and

$G_{[a b]}=2 \nabla_{[a} S_{b]}+\nabla^{c} S_{c a b}-2 S^{c} S_{c a b}$,

respectively. Note that $G_{a b}=R_{a b}-(R / 2) g_{a b}$ is the torsional analogue of the Einstein tensor (with $G_{[a b]} \neq 0$ - see Eq. (8)). Also, condition (7) ensures that the general relativistic conservation law $\nabla^{b} G_{a b}=0$ does not generally hold in the presence of torsion. Finally, taking the divergence of (8), we arrive at

$$
\begin{aligned}
\nabla^{b} G_{a b}= & \nabla^{b} G_{b a}-2\left(\nabla^{2} S_{a}-\nabla^{b} \nabla_{a} S_{b}+\nabla^{b} \nabla^{c} S_{c b a}\right) \\
& -4 \nabla^{b}\left(S^{c} S_{c a b}\right) .
\end{aligned}
$$

\subsection{Kinematics}

Introducing a timelike 4-velocity field $u_{a}$ (so that $u_{a} u^{a}=$ -1) facilitates an 1+3 decomposition of the host spacetime into time and 3-dimensional space (e.g. see [20,21]). In particular, the metric tensor splits as $g_{a b}=h_{a b}-u_{a} u_{b}$, where $h_{a b}$ is the symmetric and orthogonal to $u_{a}$ projection tensor (i.e. $h_{a b}=h_{(a b)}, h_{a b} u^{b}=0$ and $h_{a}{ }^{a}=3$ ). The kinematics of the aforementioned 4-velocity field are decoded by splitting its covariant gradient as

$\nabla_{b} u_{a}=\frac{1}{3} \Theta h_{a b}+\sigma_{a b}+\omega_{a b}-A_{a} u_{b}$.

Here, $\Theta=\mathrm{D}^{a} u_{a}$ is the volume scalar, $\sigma_{a b}=\mathrm{D}_{\langle b} u_{a\rangle}$ and $\omega_{a b}=\mathrm{D}_{[b} u_{a]}$ are respectively the shear and the vorticity tensors, while $A_{a}=\dot{u}_{a}$ is the 4 -acceleration vector. ${ }^{2}$ The volume scalar monitors the convergence/divergence of the

\footnotetext{
${ }^{2}$ Overdots indicate temporal derivatives (along the timelike $u_{a}$-field). For instance $A_{a}=\dot{u}_{a}=u^{b} \nabla_{b} u_{a}$ by definition. Spatial derivatives (orthogonal to $u_{a}$ ), on the other hand, are denoted by the covariant operator $\mathrm{D}_{a}=h_{a}{ }^{b} \nabla_{b}$. Therefore, $\Theta=\mathrm{D}^{a} u_{a}=h^{a b} \nabla_{b} u_{a}$, $\sigma_{a b}=\mathrm{D}_{\langle b} u_{a\rangle}=h_{\langle b}{ }^{d} h_{a\rangle}{ }^{c} \nabla_{d} u_{c}$, etc [20,21]. Also, round brackets denote symmetrisation and square antisymmetrisation, while angled ones indicate the symmetric and trace-free part of second rank tensors (e.g. $\sigma_{a b}=\mathrm{D}_{\langle b} u_{a\rangle}=\mathrm{D}_{(b} u_{a)}-\left(\mathrm{D}^{c} u_{c} / 3\right) h_{a b}$ by construction).
}

worldlines tangent to the $u_{a}$-field, while the shear and the vorticity describe kinematic anisotropies and rotation respectively. Finally, non-zero 4-acceleration implies that the aforementioned worldlines are not autoparallel curves.

The rate of convergence/divergence of a worldline congruence is governed by the Raychaudhuri equation. In the presence of torsion, the latter reads $[17,22]$

$$
\begin{aligned}
\dot{\Theta}= & -\frac{1}{3} \Theta^{2}-R_{(a b)} u^{a} u^{b}-2\left(\sigma^{2}-\omega^{2}\right)+\mathrm{D}_{a} A^{a}+A_{a} A^{a} \\
& +\frac{2}{3} \Theta S_{a} u^{a}-2 S_{(a b) c} u^{a} u^{b} A^{c}-2 S_{\langle a b\rangle c} \sigma^{a b} u^{c} \\
& +2 S_{[a b] c} \omega^{a b} u^{c},
\end{aligned}
$$

with only the symmetric part of the (generally asymmetric) Ricci tensor contributing to the right-hand side. Analogous propagation formulae monitor the shear and the vorticity, supplemented by three constraints relating the gradients of the kinematic variables [17].

\section{FRW-like models with torsion}

Spatially homogeneous and isotropic, Friedmann-like, spacetimes cannot naturally accommodate any arbitrary form of torsion. In what follows, we will investigate the implications of such highly symmetric torsion fields for the evolution of the cosmological spacetime.

\subsection{The torsion field}

Consider an FRW-type spacetime with non-zero torsion and a family of observers living along a timelike congruence tangent to the 4-velocity field $u_{a}$, (as defined in the previous section). The homogeneity and isotropy of the 3-dimensional rest-space of these observers, is preserved when the torsion tensor takes the form [8]

$S_{a b c}=2 \phi h_{a[b} u_{c]}$.

Due to the homogeneity of the 3 -space, $\phi$ is a scalar function of time only (i.e. $\phi=\phi(t)$ ). This torsion field also respects spatial isotropy, since the associated vector

$S_{a}=-3 \phi u_{a}, \quad$ with $\left\{\begin{array}{l}\phi>0 \Leftrightarrow S_{a} \downarrow \uparrow u_{a} \\ \phi<0 \Leftrightarrow S_{a} \uparrow \uparrow u_{a},\end{array}\right.$

is purely timelike (see also [17]). Therefore, the sign of $\phi$ fixes the relative orientation of the torsion and the 4-velocity vectors (and vice versa). In particular, $S_{a}$ is future-directed for negative $\phi$, while in the opposite case the torsion vector becomes past-directed. Finally, relations (1) and (12) give $K_{a b c}=4 \phi u_{[a} h_{b] c}$, which is the allowed form of the contortion tensor in FRW-like universes. Then, $K^{b}{ }_{a b}=-6 \phi u_{a}=$ 
$2 S_{a}=-K_{a b}{ }^{b}$ and $K^{b}{ }_{b a}=0$ as expected (see Sect. 2.1 previously).

Using the Cartan field equations (see (4) in Sect. 2.2), one can recast (12) and (13) into the following expressions

$\kappa s_{a b c}=8 \phi h_{c[a} u_{b]} \quad$ and $\quad \kappa s_{a}=12 \phi u_{a}$,

for the spin tensor and the spin vector respectively. These then combine with Eqs. (12) and (13) to guarantee that

$S_{a b c}=-\frac{1}{4} \kappa s_{c b a} \quad$ and $\quad S_{a}=-\frac{1}{4} \kappa s_{a}$.

One could use the above to replace torsion with spin in our formulae. Nevertheless, given that the two fields are simply proportional to each other, we will focus on torsion rather than spin.

\subsection{Conservation laws}

Applying relations (12) and (13) to the second of the twicecontracted Bianchi identities (see Eq. (8) in Sect. 2.2), it is straightforward to show that the right-hand side of the latter relation vanishes. This ensures that $G_{[a b]}=0$, which in turn guarantees that $R_{[a b]}=0$ and $T_{[a b]}=0$ as well. Consequently, in spatially homogeneous and isotropic spacetimes, the Ricci and the energy-momentum tensors retain their familiar (Riemannian) symmetry despite the presence of torsion. ${ }^{3}$

Introducing our form of torsion to the Einstein-Cartan field equations and using the first of the twice-contracted Bianchi identities (see Eqs. (3) and (7) respectively), leads to the constraint

$$
\nabla^{b} T_{a b}=-4 \phi\left(T_{a b} u^{b}-\kappa^{-1} \Lambda u_{a}\right),
$$

with the right-hand side vanishing for zero torsion. The high symmetry of the host spacetime demands that matter is a perfect fluid with $T_{a b}=\rho u_{a} u_{b}+p h_{a b}$, where $\rho$ and $p$ are its energy density and isotropic pressure respectively. Then, starting from (16), we obtain

$\dot{\rho}=-\Theta(\rho+p)+4 \phi\left(\rho+\kappa^{-1} \Lambda\right)$,

namely the continuity equation in Friedmann-type cosmologies with non-zero torsion. Clearly, when $\phi=0$, the above immediately reduces to the familiar general relativistic energy-density conservation law.

\footnotetext{
${ }^{3}$ In order to show the symmetry of the Ricci and energy-momentum tensors in FRW-like models with torsion, one needs to remember that 4-velocity split (see Eq. (10) in Sect. 2.3) reduces to $\nabla_{b} u_{a}=(\Theta / 3) h_{a b}$ and that $\nabla_{a} \phi=-\dot{\phi} u_{a}$ (since $\mathrm{D}_{a} \phi=0$ by default) in these highly symmetric spacetimes.
}

3.3 The Raychaudhuri equation

Spatial homogeneity and isotropy demand that $\sigma_{a b}=0=$ $\omega_{a b}=A_{a}$. Then, applying (12) and (13) to the generalised Raychaudhuri equation (see (11) in Sect. 2.3), the latter reads

$\dot{\Theta}=-\frac{1}{3} \Theta^{2}-\frac{1}{2} \kappa(\rho+3 p)+\Lambda+2 \Theta \phi$,

having also used the Einstein-Cartan equations with perfect fluid. Note the last term on the right-hand side of Eq. (18), which implies that torsion assists or inhibits the expansion/contraction of the timelike congruence tangent to the $u_{a}$-field, depending on the sign of $\phi$.

The volume scalar $(\Theta)$ of a spacetime with non-zero torsion and its purely Riemannian (i.e. torsionless) counterpart $(\tilde{\Theta})$ are related by $[17]$

$\Theta=\tilde{\Theta}+K^{a}{ }_{b a} u^{b}$,

where $K_{a b}^{b}=-6 \phi u_{a}$ (see Sect. 3.1). Consequently, the above relation simplifies to

$\Theta=\tilde{\Theta}+6 \phi=3\left(\frac{\dot{a}}{a}\right)+6 \phi=3 H\left(1+2 \frac{\phi}{H}\right)$.

Here, $\dot{a} / a=\tilde{\Theta} / 3=H$ defines the cosmological scale factor $(a=a(t))$ both in torsional and in torsion-free Friedmannian cosmologies, with $H$ being the associated Hubble parameter. ${ }^{4}$ According to (20), the divergence/convergence of worldlines in FRW-type cosmologies with torsion is not solely determined by the scale-factor evolution. Substituting (20) into Eq. (18), we obtain

$\frac{\ddot{a}}{a}=-\frac{1}{6} \kappa(\rho+3 p)+\frac{1}{3} \Lambda-2 \dot{\phi}-2\left(\frac{\dot{a}}{a}\right) \phi$.

The latter provides an alternative expression of the Raychaudhuri equation in FRW-type cosmologies with non-zero torsion, this time in terms of the model's scale factor.

\subsection{The Friedmann equations}

Treating the torsion and the metric independently, means that the line element of the spacetime is identical to its Riemannian counterpart. Therefore, in an FRW-type cosmology, we have

$$
\begin{aligned}
\mathrm{d} s^{2}= & -\mathrm{d} t^{2}+\frac{a^{2} \mathrm{~d} r^{2}}{1-K r^{2}}+a^{2} r^{2} \mathrm{~d} \vartheta^{2} \\
& +a^{2} r^{2} \sin ^{2} \vartheta \mathrm{d} \varphi^{2},
\end{aligned}
$$

with $K=0, \pm 1$ being the 3-curvature index. The above metric, together with Eq. (12) and the metricity condition (i.e. $\nabla_{c} g_{a b}=0$ ), provides the components of the generalized

\footnotetext{
${ }^{4}$ Following (20), the dimensionless ratio $\phi / H$ measures the "relative strength"of the torsion effects.
} 
connection (see Appendix A). To obtain the Ricci tensor and subsequently the Ricci scalar, we recall that

$$
R_{a b}=-\partial_{b} \Gamma_{a c}^{c}+\partial_{c} \Gamma_{a b}^{c}-\Gamma_{a c}^{e} \Gamma_{e b}^{c}+\Gamma_{a b}^{e} \Gamma_{e c}^{c}
$$

and employ a lengthy calculation (see Appendices A, B for the details). Finally, assuming a perfect fluid and involving the Einstein-Cartan field equations, we arrive at

$$
\left(\frac{\dot{a}}{a}\right)^{2}=\frac{1}{3} \kappa \rho-\frac{K}{a^{2}}+\frac{1}{3} \Lambda-4 \phi^{2}-4\left(\frac{\dot{a}}{a}\right) \phi
$$

and

$$
\frac{\ddot{a}}{a}=-\frac{1}{6} \kappa(\rho+3 p)+\frac{1}{3} \Lambda-2 \dot{\phi}-2\left(\frac{\dot{a}}{a}\right) \phi .
$$

These are the torsional analogues of the Friedmann equations, obtained here by means of metric-based techniques. Note that the last expression is identical to Eq. (21), obtained via covariant methods. Additional agreement comes by showing that the continuity equation obtained from Eqs. (24) and (25) is identical to the one derived earlier (see relation (17) in Sect. 3.2).

Following (24), torsion contributes to the total effective energy-density of the system. More specifically, the torsional analogue of the Friedmann equation recasts as

$1=\Omega_{\rho}+\Omega_{K}+\Omega_{\Lambda}+\Omega_{\phi}$,

where $\Omega_{\rho}=\kappa \rho / 3 H^{2}, \Omega_{K}=-K / a^{2} H^{2}, \Omega_{\Lambda}=\Lambda / 3 H^{2}$ and $\Omega_{\phi}=-4[1+(\phi / H)](\phi / H)$ are the associated density parameters. The strength of the torsion contribution, relative to that of the matter for example, is measured by the dimensionless ratio $\Omega_{\phi} / \Omega_{\rho}$. Note that the torsion input to the Friedmann equation vanishes when $\phi / H=0,-1$. On the other hand, torsion dominates completely when $\phi / H=-1 / 2$, which translates into $\Omega_{\phi}=1$ and vice versa. In an expanding universe (where $H>0$ ), the latter can occur only for $\phi<0$ (see also Eq. (24) earlier).

Since $q=-\ddot{a} a / \dot{a}^{2}$ defines the deceleration parameter of the universe, we may rewrite (25) as

$q H^{2}=\frac{1}{6} \kappa(\rho+3 p)-\frac{1}{3} \Lambda+2 \dot{\phi}+2 H \phi$,

Therefore, torsion can either assist or inhibit accelerated expansion. When $\phi$ is constant and negative, in particular, the presence of torsion tends to accelerate the expansion (see also Sects. 3.5.1 and 3.5.2 below).

\subsection{Characteristic solutions}

The analytical solutions presented in this section are examples aiming at demonstrating the versatility of the torsion effects upon the FRW-like host. In order to maintain the analytical nature of our treatment, as well as for reasons of mathematical simplicity and physical transparency, we will do so by assuming a simple time-invariant torsion field (i.e. one with $\phi=\phi_{0}=$ constant $) .{ }^{5}$ Nevertheless, our formalism can be readily extended to time-varying torsion as well.

Assuming zero cosmological constant and using relation (20), the continuity equation (see expression (17) in Sect. 3.2) of a barotropic medium with $p=w \rho$, reads

$\frac{\dot{\rho}}{\rho}=-3(1+w)\left(\frac{\dot{a}}{a}\right)-2(1+3 w) \phi$.

When $w=$ constant the above integrates to

$\rho=\rho_{0}\left(\frac{a}{a_{0}}\right)^{-3(1+w)} \mathrm{e}^{-2(1+3 w) \int_{t_{0}}^{t} \phi \mathrm{d} t}$,

with $\rho_{0}=\rho\left(t=t_{0}\right)$ and $a_{0}=a\left(t=t_{0}\right)$. Accordingly, the torsion effect on the energy-density evolution (carried by the exponential term on the right-hand side of the above) also depends on the matter equation of state. The torsion contribution vanishes in media with zero effective gravitational mass/energy (i.e. when $w=-1 / 3$ ). On the other hand, the energy-density evolution becomes essentially torsion dominated in the case of a vacuum stress, when $w=-1$ and $\rho=\rho_{0} \exp \left(4 \int_{t_{0}}^{t} \phi \mathrm{d} t\right)$. Therefore, $\rho=\rho(t)$ due to the presence of torsion alone, implying that media with $p=-\rho$ are not dynamically equivalent to a cosmological constant. Such a behaviour, which is in direct contrast with the purely general relativistic picture, has also been encountered in scalar-tensor theories like the Brans-Dicke theory [23-25].

\subsubsection{Vacuum and torsion-dominated solutions}

General relativistic empty FRW-type spacetimes with Euclidean spatial sections and no cosmological constant are static. The following simple example shows that torsion can change this. Indeed, when $\rho=0=K=\Lambda$, the torsional analogue of the Friedmann equation (see expression (24) in Sect. 3.4) recasts into the perfect square $(\dot{a} / a+2 \phi)^{2}=0$. The latter ensures the following relation

$\phi=\phi(t)=-\frac{1}{2} \frac{\dot{a}}{a}$,

between the torsion scalar and the cosmological scale factor. Then, the choice $\phi=\phi_{0}=$ constant leads to $\dot{a} / a=$ constant and subsequently to the de Sitter-like expansion

$a=a_{0} \mathrm{e}^{-2 \phi_{0}\left(t-t_{0}\right)}$,

when $\phi_{0}<0$. Therefore, an early universe with non-zero torsion could have undergone a phase of inflationary expansion without the need of a cosmological constant, or the presence of an inflaton field.

\footnotetext{
$\overline{5}$ It goes without saying that the same solutions also (approximately) hold in the case of slowly varying torsion.
} 
Let us now consider a vacuum spacetime with zero cosmological constant, but this time allow for hyperbolic spatial geometry. ${ }^{6}$ When $\rho=0=p=\Lambda, K=-1$ and $\phi \neq 0$, we obtain what one might call the torsion analogue of the classical Milne universe. Then, Eq. (24) factorises as $(\dot{a} / a+2 \phi+1 / a)(\dot{a} / a+2 \phi-1 / a)=0$, giving

$\phi=\phi(t)=-\frac{1}{2}\left(\frac{\dot{a}}{a} \pm \frac{1}{a}\right)$.

Setting $\phi=\phi_{0}=$ constant on the left-hand side, the above integrates to

$a=a_{0} \mathrm{e}^{-2 \phi_{0}\left(t-t_{0}\right)} \pm \frac{1}{2 \phi_{0}}\left[\mathrm{e}^{-2 \phi_{0}\left(t-t_{0}\right)} \mp 1\right]$,

giving exponential expansion when $\phi_{0}<0$. Therefore, instead of the "coasting" evolution (with $a=t$ ) of its classical counterpart, the torsional Milne universe can undergo de Sitter inflation.

Given that in empty models torsion cannot be associated with the spin of the matter, it would have to be treated as an independent, generic spacetime feature (the same also holds for the curvature). Nevertheless, to first approximation, our vacuum solutions also govern the evolution of a Friedmannlike universe with matter, provided that torsion dominates over the matter (i.e. for $\Omega_{\phi} \gg \Omega_{\rho}$ - see Eq. (26) in Sect. 3.4). Put another way, the solutions obtained in this section can be seen as limiting cases of low-density FRW-type universes with non-zero torsion.

\subsubsection{Solutions with matter}

We will now allow for matter terms in our equations, assuming Friedmann-like universes with zero 3-curvature and no cosmological constant. When dealing with pressure-free matter (i.e. dust) and torsion with $\phi=\phi_{0}=$ constant, Eqs. (24) and (25) combine to give

$\frac{\ddot{a}}{a}+\frac{1}{2}\left(\frac{\dot{a}}{a}\right)^{2}+4 \phi_{0}\left(\frac{\dot{a}}{a}\right)+2 \phi_{0}^{2}=0$.

The above accepts a solution of the form $a^{3 / 2}=\mathcal{C}_{1} \mathrm{e}^{-\phi_{0} t}+$ $\mathcal{C}_{2} \mathrm{e}^{-3 \phi_{0} t}$, with $\mathcal{C}_{1,2}$ being the integration constants. When $\phi_{0}<0$ the scale factor evolves as

$a=a_{0} \mathrm{e}^{-2 \phi_{0}\left(t-t_{0}\right)}$,

at late times. Note that we have made no a priori assumption on the relative strength of the torsion field. Taken at face value, this means that the mere presence of torsion could drive the Einstein-de Sitter universe into an accelerated regime

\footnotetext{
6 Vacuum torsional spacetimes with no cosmological constant and spherical spatial geometry do not exist in our scheme. Indeed, in such an environment Eq. (24) recasts into $(\dot{a} / a+2 \phi)^{2}=-1 / a^{2}$, which is impossible.
}

analogous to that of the de Sitter model. Although it seems premature to claim that torsion can provide a viable alternative answer to the inflaton field and/or dark energy, analogous claims have been made by $[12,13]$.

False-vacuum cosmologies have been typically associated with inflation, being the driving force of the exponential expansion. In what follows, we will consider torsional FRWlike universes with false-vacuum barotropic index $w=-1$, Euclidean spatial geometry and zero cosmological constant. Then, on using (29), the associated Friedmann equation (see (24) in Sect. 3.4) recasts into

$H=-2 \phi \pm \sqrt{\frac{\kappa \rho_{0}}{3}} \mathrm{e}^{2 \int_{t_{0}}^{t} \phi \mathrm{d} t}$.

Torsion ensures that the Hubble parameter varies in time, in contrast to the torsionless case where $H=$ constant. Recalling that $H=\dot{a} / a$ and setting $\phi=\phi_{0}=$ constant, Eq. (36) integrates to

$a=a_{0} \mathrm{e}^{-2 \phi_{0}\left(t-t_{0}\right) \pm \frac{1}{2 \phi_{0}} \sqrt{\frac{\kappa \rho_{0}}{3}}\left[\mathrm{e}^{2 \phi_{0}\left(t-t_{0}\right)}-1\right]}$.

When $\phi_{0}<0$ the above asymptotically reduces to solution (31) at late times. For $\phi_{0}>0$, on the other hand, the latetime evolution of the scale factor also allows for exponential "deflation". 7

The solutions presented in Sects. 3.5.1 and 3.5.2 are characteristic of the versatile and the occasionally nontrivial implications of the torsion field, even when the latter takes the very restricted form imposed by the high symmetry of the Friedmann-like host.

\section{Observational bounds on cosmic torsion}

The literature contains a number of proposals for observational tests of torsion, the majority of which work within the realm of our solar system [1-7]. Here, we will attempt to put cosmological bounds on the torsion field, by exploiting the fact that it "gravitates" and therefore modifies the expansion dynamics of the host universe.

\subsection{Steady-state torsion}

A measure of the torsion contribution to the expansion kinematics is given by the dimensionless ratio $\phi / H$. Then, setting $K=0=\Lambda$ and $\lambda=\phi / H$, the Friedmann and the continuity

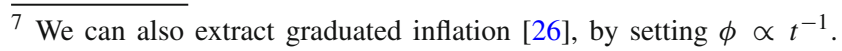
Indeed, substituted into Eq. (24), this choice leads to

$a=a_{0}\left(\frac{t}{t_{0}}\right)^{-2 \phi_{0} t_{0}} \mathrm{e}^{ \pm \sqrt{\frac{\kappa \rho_{0} t_{0}^{2}}{3\left(2 \phi_{0} t_{0}+1\right)^{2}}}\left(\frac{t}{t_{0}}\right)^{2 \phi_{0} t_{0}+1}}$. 
equations (see (24) and (28)) become

$$
H^{2}=\frac{\kappa \rho}{3(2 \lambda+1)^{2}}
$$

and

$\frac{\dot{\rho}}{\rho}=-[3+2 \lambda+3 w(1+2 \lambda)] H$,

respectively (with $\lambda \neq-1 / 2$ ). ${ }^{8}$ The former relation shows that torsion changes the Hubble-flow rate, which means that it can affect physical interactions that are sensitive to the pace of the cosmic expansion, like the Big-Bang Nucleosynthesis (BBN) of helium-4 for example (see Sect. 4.2 next).

When the $\lambda$-ratio decays over time, the effects of torsion on Eq. (40) fade away. In the opposite case, on the other hand, the right-hand side of the continuity equation becomes torsiondominated. Here, we will take the middle path and assume "steady-state" torsion with $\lambda=\phi / H=$ constant. In other words, we will assume that the torsion input to the universal expansion (see Eq. (20) in Sect. 3.3) does not change in time. Then, given that $H=\dot{a} / a$, the above two relations combine to the solution

$a=a_{0}\left(\frac{t}{t_{0}}\right)^{2 /[3+2 \lambda+3 w(1+2 \lambda)]}$.

Next, we will employ this result to impose cosmological bounds on $\lambda$ and on torsion itself. In doing so, we will turn to the early universe and to the epoch of primordial nucleosynthesis specifically.

\section{2 $\mathrm{BBN}$ bounds on torsion}

In the early radiation era of the universe $w=1 / 3$ and solution (41) reduces to $a \propto t^{1 / 2(1+\lambda)}$, with $\lambda \neq-1$. Recall that, when $\lambda=\phi / H=-1$, the torsion input to the Friedmann equation vanishes (see expression (26) in Sect. 3.4). The above, together with Eq. (39), lead to the following expression for the radiation energy density

$\rho_{(\gamma)}=\frac{3(1+2 \lambda)}{4 \kappa(1+\lambda)^{2} t^{2}}=\sigma T^{4}$

where $\sigma$ is the black-body constant. The presence of $\lambda$ means that the temperature evolution differs from that of the standard (torsionless) Friedmann universe (with three light neutrino species).

The freeze-out temperature $\left(T_{f r}\right)$ of the neutron-proton kinetic equilibrium occurs when the weak interaction rate $\left(\Gamma_{w k} \propto T^{5}\right)$ for the neutron-proton exchanges $(n \leftrightarrow p+$ $e^{-}+\bar{v}_{e}, n+v_{e} \leftrightarrow p+e^{-}$and $\left.n+e^{+} \leftrightarrow p+\bar{v}_{e}\right)$ equals the Hubble rate. This yields a simple analytic expression for

\footnotetext{
${ }^{8}$ When $\lambda=-1 / 2$ we have $\Omega_{\phi}=1$ (see Eq. (26)), corresponding to a purely torsional (empty) FRW-like universe.
}

the ratio of the freeze-out temperatures between cosmologies with torsion $\left(T_{f r} \equiv T_{f r}(\lambda \neq 0)\right)$ and torsionless ones $\left(\tilde{T}_{f r} \equiv T_{f r}(\lambda=0)\right)$. In particular, we find that

$\frac{\tilde{T}_{f r}}{T_{f r}}=(1+2 \lambda)^{1 / 3}$.

When $\lambda>0$ torsion will reduce the freeze-out temperature. Then, the neutron-to-proton ratio $(\mathcal{N}=n / p)$ will freezein at lower temperatures and the residual helium-4 abundance will decrease compared to that in the standard (torsionfree) Friedmann universe. The slowing of the expansion rate allows neutrons and protons to remain in non-relativistic kinetic equilibrium down to lower temperatures, with fewer neutrons per proton surviving before the equilibrium is broken at $T_{f r}$.

The above torsion effect provides a very rare (if not unique) example of a modified early-universe model with a reduced helium-4 abundance. All other common modifications (i.e. extra light neutrino species, magnetic fields, anisotropies, Brans-Dicke fields, etc) lead to higher freezeout temperatures. This increases the frozen-in $n / p$ ratio and therefore enhances the residual abundance of helium-4. In the presence of torsion this happens when $\lambda<0$.

We may quantify these arguments by recalling that the frozen-in neutron-to-proton ratio is $\mathcal{N}_{f r}=\exp \left(-\Delta / T_{f r}\right)$, where $\Delta$ measures the neutron-proton mass difference and we have set $\sigma=1$. Following (43), we then arrive at the following simple relation

$\mathcal{N}_{f r}=\tilde{\mathcal{N}}_{f r}^{(1+2 \lambda)^{1 / 3}}$,

between the torsional and the torsionless freeze-in ratios. In the standard torsion-free universe, $\tilde{\mathcal{N}} \simeq 1 / 5$ at freeze-out, but falls to $\tilde{\mathcal{N}} \simeq 1 / 7$ at nucleosynthesis due to free neutron beta-decay. This means that the residual mass fraction of the synthesised helium- 4 is $\tilde{Y}=2 \tilde{\mathcal{N}}_{n s} /\left(1+\tilde{\mathcal{N}}_{n s}\right) \simeq 0.25$. Assuming "weak"torsion with $|\lambda|=|\phi| / H<1$ and keeping in mind that $\tilde{\mathcal{N}}_{n s}, \mathcal{N}_{n s}<1$, we obtain

$$
\begin{aligned}
Y & =\frac{2 \mathcal{N}_{n s}}{1+\mathcal{N}_{n s}} \simeq \mathcal{N}_{n s} \simeq \tilde{\mathcal{N}}_{n s}^{1+2 \lambda / 3} \\
& =\tilde{Y} \tilde{\mathcal{N}}_{n s}^{2 \lambda / 3} \simeq 0.25 \times 7^{-2 \lambda / 3}
\end{aligned}
$$

Recent observational evidence for the allowed range of the primordial helium-4 abundance (extrapolated to zero metals) yields the range $0.2409 \lesssim Y \lesssim 0.2489$. Now combining the standard zero-torsion prediction of $Y \simeq 0.24703$ [27] with the observed range in Eq. (45), leads to a strong observational constraint on the torsion parameter:

$-0.005813 \lesssim \lambda \lesssim+0.019370$

which is consistent with our $|\phi| / H<1$ assumption. 


\section{Static spacetimes with torsion}

The extra degrees of freedom that torsion introduces are expected to relax some of the standard constraints associated with static spacetimes. We will therefore now turn our attention to the study of static (homogeneous and isotropic) models with torsion.

\subsection{The Einstein-static analogue}

Static spacetimes with non-zero torsion have been studied in the past, assuming matter in the form of the Weyssenhoff fluid [28]. The latter, however, is incompatible with the high symmetry of the FRW-like models and the Einstein static universe as well. For this reason, an unpolarised spin field was adopted, with a spin tensor that averages to zero (e.g. see [29-31]). Here, instead, we address the FRW-compatibility issue by adopting a form for the torsion/spin fields that is compatible with the spatial isotropy and homogeneity of the Friedmannian spacetimes (see Eqs. (12) and (15) in Sect. 3.1 earlier).

In static environments the absence of evolution means that $\dot{a}=0=\ddot{a}$ and $\dot{\rho}=0=\dot{p}=\dot{\phi}$. Then, the Friedmann equations derived in Sect. 3.4 assume the (static) form

$$
\frac{1}{3} \kappa \rho_{0}-\frac{K}{a_{0}^{2}}+\frac{1}{3} \Lambda-4 \phi_{0}^{2}=0
$$

and

$$
\frac{1}{2} \kappa\left(\rho_{0}+3 p_{0}\right)-\Lambda=0
$$

where $\rho_{0}, p_{0}$ and $\phi_{0}$ are constants. It follows that for ordinary matter (with $\rho_{0}>0$ and $\rho_{0}+3 p_{0}>0$ ), the static solution requires the presence of a positive cosmological constant, just like in the conventional Einstein-static universe (see Eq. (48)). However, the torsional analogue of the Einsteinstatic model does not necessarily need positive spatial curvature. Indeed, expression (47) guarantees that torsion can play the role of the positive curvature and that the 3-curvature index can take all the available values (i.e. $K=0, \pm 1$ ). Also, for matter with vanishing total gravitational energy, namely when $\rho_{0}+3 p_{0}=0$, there is a static solution with Euclidean spatial hypersurfaces, zero cosmological constant, but nonzero torsion (i.e. $K=0=\Lambda$ and $\rho_{0}, \phi_{0} \neq 0$ ).

Additional constraints come after successively eliminating the cosmological constant and the matter density from the set of (47) and (48). In particular, we arrive at the following expressions

$$
\frac{1}{2} \kappa \rho_{0}(1+w)-\frac{K}{a_{0}^{2}}=4 \phi_{0}^{2}
$$

and

$$
\frac{(1+w) \Lambda}{1+3 w}-\frac{K}{a_{0}^{2}}=4 \phi_{0}^{2},
$$

between the variables of the static model (with $w=p_{0} / \rho_{0}$ ). Note that the last two constraints combine to reproduce (48). Finally, when $K=+1$, condition (49) gives

$a_{0}=\sqrt{\frac{2}{\kappa \rho_{0}(1+w)-8 \phi_{0}^{2}}}$,

with $\kappa \rho_{0}(1+w)>8 \phi_{0}^{2}$. Therefore, keeping the energy density of the matter fixed, the introduction of torsion increases the radius of the Einstein-static universe. Put another way, a torsional Einstein universe should be larger in size than its classic counterpart. A closely analogous effect, though in that case expressed in terms of the spin, was observed by [32].

\subsection{Stability of the static model}

We will test the stability of the static model, by looking at the evolution of linear conformal perturbations (i.e. into other FRW models so that $\phi$ is not inhomogeneously perturbed). In particular, assuming that $\phi=\phi_{0}=$ constant at the linear level, we consider deviations of the form

$a=a_{0}+\delta a, \quad \rho=\rho_{0}+\delta \rho$ and $\quad p=p_{0}+\delta p$,

where $\delta a \ll a_{0}, \delta \rho \ll \rho_{0}$ and $\delta p \ll p_{0}$. Substituting these into Eqs. (24) and (25), we solve (24) for the matter density. Then, using the resulting expression in (25), employing the background relations (47)-(50), setting $\delta=\delta a / a_{0} \ll 1$, and keeping up to first-order terms, leads to the differential equation,

$a_{0}^{2} \ddot{\delta}+2(2+3 w) \phi_{0} a_{0}^{2} \dot{\delta}-(1+3 w) K \delta=0$,

for the linear evolution of the scale-factor perturbation. Assuming matter with zero pressure and Euclidean spatial sections, namely setting $w=0=K$, the solution reads

$\delta=\mathcal{C}_{1}+\mathcal{C}_{2} \mathrm{e}^{-4 \phi_{0} t}$

with $\mathcal{C}_{1,2}$ being the integration constants. This result shows (neutral) stability when $\phi_{0}>0$ and (exponential) instability for $\phi_{0}<0$. One can also show that (53) leads to essentially the same solution, when matter satisfies the strong energy condition (i.e. for $1+3 w>0$ ). Hence, an Einstein-static universe with torsion, conventional matter and zero 3-curvature is stable when the (timelike) torsion vector is past-directed, but unstable when $S_{a}$ is future-directed (see (13) in Sect. 3.1).

If we allow the 3-dimensional surfaces to have non-zero curvature, but maintain our assumption of pressureless matter (i.e. $K= \pm 1$ and $w=0$ ), Eq. (53) solves to give

$\delta=\mathcal{C}_{1} \mathrm{e}^{\alpha_{1} t}+\mathcal{C}_{2} \mathrm{e}^{\alpha_{2} t}$, 
where

$\alpha_{1,2}=-2 \phi_{0} \pm \sqrt{4 \phi_{0}^{2}+\frac{1}{a_{0}^{2}}}$

and

$\alpha_{1,2}=-2 \phi_{0} \pm \sqrt{4 \phi_{0}^{2}-\frac{1}{a_{0}^{2}}}$,

when $K=+1$ and $K=-1$ respectively. In the former case, solution (55), (56) contains at least one (exponentially) growing mode, regardless of the sign of $\phi_{0}$ (i.e. of the orientation of the torsion vector). Note also that the nature of the solution does not change so long as $1+3 w>0$. Therefore, an Einstein-static universe with non-zero torsion, conventional matter and positively curved spatial hypersurfaces is always unstable. In models with negative 3-curvature the evolution is more involved. Following (55) and (57), for $\phi_{0}^{2} a_{0}^{2}>1 / 4$, we have stability when $\phi_{0}>0$ and instability for $\phi_{0}<0$. When $\phi_{0}^{2} a_{0}^{2}<1 / 4$, on the other hand, the solution of Eq. (55) contains an imaginary part. This translates into an oscillation with amplitude $\delta \propto \mathrm{e}^{-2 \phi_{0} t}$. As before, the nature of the solution does not change so long as $1+3 w>0$. Thus, an Einstein-static universe with non-zero torsion, conventional matter and open spatial hypersurfaces can be stable provided that $\phi_{0}>0$. An alternative method of testing the linear stability of the static solution, which arrives at the same conclusions but provides a different view of the issue, is given in Appendix C.

Our stability analysis assumed homogeneous linear perturbations, similar to those employed by Eddington in his classic study of Einstein's static world [33]. This implies that the stable configurations reported here may prove unstable when inhomogeneous perturbations of all the three possible types (i.e. scalar, vector and tensor) are accounted for (see [34] for such a linear-stability analysis on the classic Einstein-static spacetime). ${ }^{9}$

\section{Discussion}

Allowing for an asymmetric affine connection provides the simplest classical extension of general relativity, by incorporating the effects of spacetime torsion into the theory. The latter can then be use to study a variety of theoretical problems, ranging from singularity theorems and cosmology, to supergravity and quantum gravity (e.g. see [40] and references therein). However, torsion and spin are generally

\footnotetext{
9 The linear stability of the Einstein static universe against specific types of perturbations was also investigated by [35-37]. There are also stability studies against general Mixmaster spatially homogeneous modes [38] and of static ghost models [39].
}

incompatible with the high symmetry of the FRW cosmologies, which means that one needs to consider torsion/spin fields that preserve both the homogeneity and the isotropy of these universes. Here, we have addressed this issue by adopting a specific profile for the torsion tensor that belongs to the class of the vectorial torsion fields and it is monitored by a single scalar function of time $[8,11]$. Nevertheless, even this highly constraint form of torsion was found capable of drastically altering the standard evolution of the classic FRW cosmologies.

Using $1+3$ covariant and metric-based techniques, we derived the associated continuity, Friedmann and Raychaudhuri equations. These allowed us to quantify the relative strength of the torsion effects by means of an associated $\Omega$-parameter. A number of new possibilities emerged. We found that torsion can play the role of the spatial curvature and mimic the effects of the cosmological constant, depending on the specifics of the scenario in hand. The orientation of the torsion vector, relative to the fundamental 4-velocity field, was a decisive factor, since it determines whether torsion will tend to decelerate or accelerate the expansion of the host spacetime. Empty spacetimes with zero 3-curvature, no cosmological constant and non-zero torsion are not necessarily static, but can experience exponential expansion (see also [22] for similar results). The introduction of spatial curvature, or matter, did not seem to change the aforementioned picture. So, in the presence of torsion, the Einstein-de Sitter universe can experience exponential de Sitter-like inflation. ${ }^{10}$ All these findings raise the possibility that universes with non-zero torsion might have gone through an early (or a late) phase of accelerated expansion without requiring a cosmological constant, an inflaton field, or some sort of dark energy.

Looking for potentially observable cosmological signatures of torsion, we considered its effects on primordial nucleosynthesis. We found that torsion can increase, as well as decrease, the production of helium-4, by changing the expansion rate of the universe at the time of primordial nucleosynthesis. Using our solution for a radiation-dominated Friedmann universe, we were able to calculated the expected abundance of helium-4 from primordial nucleosynthesis when torsion is present. Combining these results with the observationally allowed range of the helium- 4 abundance, we were able to impose strong constraints on the relative strength of the torsion field.

Our study also found that there exist Einstein-static universes with torsion that are not closed, but can have all three

\footnotetext{
10 These results were obtained after assuming a time-invariant torsion field. Nevertheless, our formalism can be readily extended to include time-varying torsion (e.g. see footnote 7 and Sect. 4.1), which is what we intent to do in future work. In that case, one might also have to go beyond the analytical treatment and use numerical techniques as well.
} 
types of spatial curvature. Unlike the classic (torsion-free) Einstein model, for appropriate choices of the torsion field and of the spatial curvature, these static universes can be stable against linear scalar perturbations even for pressureless (dust) matter. Overall, despite the restrictions imposed by their high symmetry, FRW-like universes with torsion exhibit a rich phenomenology that could distinguish them from their general-relativistic counterparts.

Acknowledgements We would like to thank Peter Stichel for drawing our attention to reference [8]. CGT acknowledges support from a visiting fellowship by Clare Hall College and visitor support by DAMTP at the University of Cambridge. JDB was supported by the Science and Technology Facilities Council (STFC) of the UK.

Data Availability Statement This manuscript has no associated data or the data will not be deposited. [Authors' comment: There is no data associated with our paper. It is a theoretical paper and no experimental data was used.]

Open Access This article is distributed under the terms of the Creative Commons Attribution 4.0 International License (http://creativecomm ons.org/licenses/by/4.0/), which permits unrestricted use, distribution, and reproduction in any medium, provided you give appropriate credit to the original author(s) and the source, provide a link to the Creative Commons license, and indicate if changes were made.

Funded by SCOAP S $^{3}$.

\section{Appendix}

\section{A The generalised connection}

Assuming that the indices $0,1,2$ and 3 correspond to the coordinates $t, r, \vartheta$ and $\varphi$ respectively, the Christoffel symbols of a FRW-type spacetime are (e.g. see [41])

$\tilde{\Gamma}_{11}^{0}=\frac{a \dot{a}}{1-K r^{2}}, \quad \tilde{\Gamma}_{22}^{0}=a \dot{a} r^{2}, \quad \tilde{\Gamma}_{33}^{0}=a \dot{a} r^{2} \sin ^{2} \vartheta$,

$\tilde{\Gamma}^{1}{ }_{01}=\tilde{\Gamma}_{10}^{1}=\frac{\dot{a}}{a}, \quad \tilde{\Gamma}_{11}^{1}=\frac{K r}{1-K r^{2}}$,

$\tilde{\Gamma}_{22}^{1}=-r\left(1-K r^{2}\right), \quad \tilde{\Gamma}_{33}^{1}=-r\left(1-K r^{2}\right) \sin ^{2} \vartheta$,

$\tilde{\Gamma}_{02}^{2}=\tilde{\Gamma}_{20}^{2}=\frac{\dot{a}}{a}, \quad \tilde{\Gamma}_{12}^{2}=\tilde{\Gamma}_{21}^{2}=\frac{1}{r}$,

$\tilde{\Gamma}_{33}^{2}=-\cos \vartheta \sin \vartheta$

$\tilde{\Gamma}^{3}{ }_{03}=\tilde{\Gamma}_{30}^{3}=\frac{\dot{a}}{a}, \quad \tilde{\Gamma}_{13}^{3}=\tilde{\Gamma}_{31}^{3}=\frac{1}{r}$,

$\tilde{\Gamma}^{3}{ }_{23}=\tilde{\Gamma}^{3}{ }_{32}=\cot \vartheta$.

Combining (12) with the above, the non-zero components of the torsion tensor are

$S^{1}{ }_{01}=S^{2}{ }_{02}=S^{3}{ }_{03}=\phi$

and

$S^{1}{ }_{10}=S_{20}^{2}=S_{30}^{3}=-\phi$.
Putting (58)-(60) together and recalling that $\Gamma^{a}(b c)=$ $\tilde{\Gamma}^{a} b c+2 S_{(b c)}{ }^{a}$ (see Sect. 2.1), we find

$\Gamma_{(11)}^{0}=\frac{a \dot{a}+2 \phi a^{2}}{1-K r^{2}}, \quad \Gamma_{(22)}^{0}=r^{2}\left(a \dot{a}+2 \phi a^{2}\right)$,

$\Gamma_{(33)}^{0}=r^{2} \sin ^{2} \vartheta\left(a \dot{a}+2 \phi a^{2}\right)$,

$\Gamma_{(01)}^{1}=\frac{\dot{a}}{a}+\phi, \quad \Gamma_{(11)}^{1}=\frac{K r}{1-K r^{2}}$,

$\Gamma_{(22)}^{1}=-r\left(1-K r^{2}\right), \quad \Gamma_{(33)}^{1}=-r\left(1-K r^{2}\right) \sin ^{2} \vartheta$,

$\Gamma_{(02)}^{2}=\frac{\dot{a}}{a}+\phi, \quad \Gamma_{(12)}^{2}=\frac{1}{r}, \quad \Gamma_{(33)}^{2}=-\cos \vartheta \sin \vartheta$,

$\Gamma_{(03)}^{3}=\frac{\dot{a}}{a}+\phi, \quad \Gamma_{(13)}^{3}=\frac{1}{r}, \quad \Gamma_{(23)}^{3}=\cot \vartheta$.

Finally, noting that $\Gamma^{a}{ }_{b c}=\Gamma^{a}{ }_{(b c)}+S^{a}{ }_{b c}$ and using the auxiliary results (60) and (61), we evaluate the non-zero components of the asymmetric affine connection

$\Gamma_{11}^{0}=\frac{a \dot{a}+2 \phi a^{2}}{1-K r^{2}}, \quad \Gamma_{22}^{0}=r^{2}\left(a \dot{a}+2 \phi a^{2}\right)$,

$\Gamma_{33}^{0}=r^{2} \sin ^{2} \vartheta\left(a \dot{a}+2 \phi a^{2}\right)$,

$\Gamma^{1}{ }_{01}=\frac{\dot{a}}{a}+2 \phi, \quad \Gamma^{1}{ }_{10}=\frac{\dot{a}}{a}, \quad \Gamma^{1}{ }_{11}=\frac{K r}{1-K r^{2}}$,

$\Gamma_{22}^{1}=-r\left(1-K r^{2}\right), \quad \Gamma_{33}^{1}=-r\left(1-K r^{2}\right) \sin ^{2} \vartheta$,

$\Gamma_{02}^{2}=\frac{\dot{a}}{a}+2 \phi, \quad \Gamma^{2}{ }_{20}=\frac{\dot{a}}{a}, \quad \Gamma^{2}{ }_{12}=\Gamma^{2}{ }_{21}=\frac{1}{r}$,

$\Gamma^{2}{ }_{33}=-\cos \vartheta \sin \vartheta$

$\Gamma_{03}^{3}=\frac{\dot{a}}{a}+2 \phi, \quad \Gamma^{3}{ }_{30}=\frac{\dot{a}}{a}, \quad \Gamma^{3}{ }_{13}=\Gamma_{31}^{3}=\frac{1}{r}$,

$\Gamma^{3}{ }_{23}=\Gamma_{32}^{3}=\cot \vartheta$.

\section{B Ricci tensor and Friedmann equations}

The Ricci curvature tensor has been expressed in terms of the generalised (asymmetric) affine connection in Sect. 3.4 - see Eq. (23) there. The latter, together with (62), leads to

$$
\begin{aligned}
R_{00}= & -3\left[\frac{\ddot{a}}{a}+2 \dot{\phi}+2 \phi\left(\frac{\dot{a}}{a}\right)\right], \\
R_{11}= & \frac{2 a^{2}}{1-K r^{2}}\left[\frac{\ddot{a}}{2 a}+\dot{\phi}+\left(\frac{\dot{a}}{a}\right)^{2}+5 \phi \frac{\dot{a}}{a}+4 \phi^{2}+\frac{K}{a^{2}}\right], \\
R_{22}= & 2 a^{2} r^{2}\left[\frac{\ddot{a}}{2 a}+\dot{\phi}+\left(\frac{\dot{a}}{a}\right)^{2}+5 \phi \frac{\dot{a}}{a}+4 \phi^{2}+\frac{K}{a^{2}}\right], \\
R_{33}= & 2 a^{2} r^{2} \sin ^{2} \vartheta \\
& \times\left[\frac{\ddot{a}}{2 a}+\dot{\phi}+\left(\frac{\dot{a}}{a}\right)^{2}+5 \phi \frac{\dot{a}}{a}+4 \phi^{2}+\frac{K}{a^{2}}\right]
\end{aligned}
$$


Given that $R_{a}{ }^{b}=g^{b c} R_{a c}$, the mixed components of the Ricci tensor are

$R_{0}^{0}=3\left[\frac{\ddot{a}}{a}+2 \dot{\phi}+2 \phi\left(\frac{\dot{a}}{a}\right)\right]$

and

$$
\begin{aligned}
R_{1}{ }^{1} & =2\left[\frac{\ddot{a}}{2 a}+\dot{\phi}+\left(\frac{\dot{a}}{a}\right)^{2}+5 \phi \frac{\dot{a}}{a}+4 \phi^{2}+\frac{K}{a^{2}}\right] \\
& =R_{2}{ }^{2}=R_{3}{ }^{3} .
\end{aligned}
$$

Consequently, the Ricci scalar of an FRW-like spacetime with non-zero torsion reads

$R=6\left[\frac{\ddot{a}}{a}+\left(\frac{\dot{a}}{a}\right)^{2}+\frac{K}{a^{2}}+2 \dot{\phi}+6 \phi\left(\frac{\dot{a}}{a}\right)+4 \phi^{2}\right]$.

Also, the energy-momentum tensor of a perfect fluid takes the diagonal form $T_{a}^{b}=\operatorname{diag}[-\rho, p, p, p]$. Plugging all of the above into the Einstein-Cartan field equations (see (3) in Sect. 2.2), we arrive at

$$
\left(\frac{\dot{a}}{a}\right)^{2}=\frac{1}{3} \kappa \rho-\frac{K}{a^{2}}+\frac{1}{3} \Lambda-4 \phi\left(\frac{\dot{a}}{a}\right)-4 \phi^{2},
$$

and

$$
\frac{\ddot{a}}{a}=-\frac{1}{6} \kappa(\rho+3 p)+\frac{1}{3} \Lambda-2 \dot{\phi}-2 \phi\left(\frac{\dot{a}}{a}\right) .
$$

These are the Friedmann equations of an FRW-type cosmology with torsion (see Sect. 3.4 earlier).

\section{Linear stability of the static solution}

We will employ the linear-stability technique of ordinary differential equations to the static model of Sect. 5.1. In doing so, we first recast Eq. (53) into the first-order system

$\dot{x}=f(x, y)=y$

and

$\dot{y}=g(x, y)=\mu y+v x$,

with $x=\delta, \mu=-2(2+3 w) \phi_{0}$ and $v=(1+3 w) K / a_{0}^{2}$. The Jacobian matrix of the above is

$$
\left(\begin{array}{ll}
\partial f / \partial x & \partial f / \partial y \\
\partial g / \partial x & \partial g / \partial y
\end{array}\right)_{0}=\left(\begin{array}{ll}
0 & 1 \\
v & \mu
\end{array}\right),
$$

where the zero suffix denotes the static solution (with $(x=$ $0, y=0)$ and $f(0,0)=0=g(0,0)$ ). The eigenvalues of matrix (71) are the roots of the associated characteristic polynomial, namely

$\lambda_{1,2}=\frac{1}{2}\left(\mu \pm \sqrt{\mu^{2}+4 v}\right)$.
For simplicity, but without compromising generality, let us confine to the case of pressure-free matter (with $w=0$ - see also Sect. 5.2 earlier). Then, the eigenvalues reduce to

$\lambda_{1,2}=-2 \phi_{0} \pm \sqrt{4 \phi_{0}^{2}+\frac{K}{a_{0}^{2}}}$.

When the spatial hypersurfaces have spherical geometry, the quantity inside the square root is always positive, in which case both eigenvalues are real. More specifically, $\lambda_{1}$ is positive and $\lambda_{2}$ is negative, implying that the static solution is a saddle point. For hyperbolic spatial surfaces, the two eigenvalues are real provided that $a_{0}^{2} \phi_{0}^{2}>1 / 4$. Then, both $\lambda_{1}$ and $\lambda_{2}$ are positive when $\phi_{0}<0$, while for $\phi_{0}>0$ the two eigenvalues are negative. In the former case the static solution is unstable, but in the latter is stable. Alternatively, when $a_{0}^{2} \phi_{0}^{2}<1 / 4$, the eigenvalues are both complex. In particular, $\operatorname{Re}\left(\lambda_{1}\right)>0$ and $\operatorname{Re}\left(\lambda_{2}\right)>0$ for $\phi_{0}<0$, which implies instability. For $\phi_{0}>0$, on the other hand, we have $\operatorname{Re}\left(\lambda_{1,2}\right)<0$ and stability. The same is also true when $a_{0}^{2} \phi_{0}^{2}=1 / 4$.

Note that the above analysis does not apply to spacetimes with Euclidean spatial sections (i.e. when $K=0$ ), because then the first of the two eigenvalues will vanish.

\section{References}

1. R.T. Hammond, Rep. Prog. Phys. 65, 599 (2002)

2. Y. Mao, M. Tegmark, A.H. Guth, S. Cabi, Phys. Rev. D 76, 104029 (2007)

3. V.A. Kostelecky, N. Russell, J. Tasson, Phys. Rev. Lett. 100, 111102 (2008)

4. R. March, G. Bellettini, R. Tauraso, S. Dell'Angello, Phys. Rev. D 83, 104008 (2011)

5. F.W. Hehl, Y.N. Obukhov, D. Puetzfeld, Phys. Lett. A 377, 1775 (2013)

6. D. Puetzfeld, Y.N. Obukhov, Int. J. Mod. Phys. D 23, 1442004 (2014)

7. R.-H. Lin, X.H. Zhai, X.Z. Li, Eur. Phys. J. C 77, 504 (2017)

8. M. Tsamparlis, Phys. Rev. D 24, 1451 (1981)

9. G.J. Olmo, Int. J. Mod. Phys. D 20, 413 (2011)

10. S. Capozzielo, R. Cianci, C. Stornaiolo, S. Vignolo, Phys. Scr. 78, 065010 (2008)

11. Jimenez J. Beltran, T.S. Koivisto, Phys. Lett. B 756, 400 (2016)

12. N.J. Poplawski, Phys. Lett. B 694, 181 (2010)

13. N.J. Poplawski, Astron. Rev. 8, 108 (2013)

14. A.N. Ivanov, M. Wellenzohn, Astrophys. J. 829, 47 (2016)

15. S. Akhshabi, E. Qorani, F. Khajenabi, Europhys. Lett. 119, 29002 (2017)

16. R. Banerjee, S. Chakraborty, P. Mukherjee, Phys. Rev. D 98, 083506 (2018)

17. K. Pasmatsiou, C.G. Tsagas, J.D. Barrow, Phys. Rev. D 95, 104007 (2017)

18. F.W. Hehl, P. von der Heyde, G.D. Kerlick, Rev. Mod. Phys. 48, 373 (1976)

19. F.W. Hehl, Y.N. Obukhov, Ann. Fond. Broglie 32, 157 (2007)

20. C.G. Tsagas, A. Challinor, R. Maartens, Phys. Rep. 465, 61 (2008)

21. G.F.R. Ellis, R. Maartens, M.A.H. MacCallum, Relativistic Cosmology (Cambridge University Press, Cambridge, 2012) 
22. D. Iosifidis, C.G. Tsagas, A.C. Petkou, Phys. Rev. D 98, 104037 (2018)

23. H. Nariai, Prog. Theor. Phys. 40, 48 (1969)

24. C. Mathiazhagen, V.B. Johri, Class. Quantum Gravity 1, L29 (1984)

25. J.D. Barrow, K.-I. Maeda, Nucl. Phys. B 341, 294 (1990)

26. J.D. Barrow, Phys. Lett. B 235, 40 (1990)

27. R.H. Cyburt, B.D. Fields, K.A. Olive, T.-H. Yeh, Rev. Mod. Phys. 88, 015004 (2016)

28. J. Weyssenhoff, J. Raade, Acta Phys. Polon. 9, 7 (1947)

29. B. Kuchowicz, Gen. Relativ. Gravit. 9, 511 (1978)

30. M. Gasperini, Phys. Rev. Lett. 56, 2873 (1986)

31. K. Atazadeh, JCAP 06, 020 (2014)

32. C.G. Böhmer, Class. Quantum Gravity 21, 1119 (2004)

33. A.S. Eddington, Mon. Not. R. Astron. Soc. 90, 668 (1930)
34. J.D. Barrow, G.F.R. Ellis, R. Maartens, C.G. Tsagas, Class. Quantum Gravity 20, L155 (2003)

35. E.R. Harrison, Rev. Mod. Phys. 39, 862 (1967)

36. G.W. Gibbons, Nucl. Phys. B 292, 784 (1987)

37. G.W. Gibbons, Nucl. Phys. B 310, 636 (1988)

38. J.D. Barrow, K. Yamamoto, Phys. Rev. D 85, 083505 (2012)

39. J.D. Barrow, C.G. Tsagas, Class. Quantum Gravity 26, 195003 (2009)

40. M. Blagojevic, F.W. Hehl, Gauge Theories and Gravitation (Imperial College Press, London, 2013)

41. J.V. Narlikar, Introduction to Cosmology (Jones and Bartlett, Boston, 1983) 